\section{Rise in Child and Adolescent Emergency Psychiatric Presen- tations: Is it an Evolving Chal- lenge for Mental Health Professionals?}

Sir,

Child and adolescent mental health problems have always been an area of interest for professionals from all the psychiatric sub-specialties. It is believed that more than half of those, attending adult psychiatry clinics have mental health problems from their childhood. ${ }^{1}$ Liaison psychiatry is an interesting field, offering a varied exposure to psychiatric presentations from all age groups. The emergency department is one of the main hubs for liaison psychiatry with peoplefrom all age groups, including the adolescent population. We have closely monitored child and adolescent psychiatric presentations in the emergency department of our teaching hospital, observing a rise in these presentations in recentyears.

The rise in emotional and psychological problems in adolesecnts is not merely a concernn for child and adolescent mental health specialists (CAMHs), but potentially an issue for adult mental health services, addiction services or even forensic services. ${ }^{2}$ Therefore, early intervention and treatment could not only reduce the disease burden but also make a positive impact on lives of patients and their families. ${ }^{3}$ We had 26 ED mental health presentations of adolescent population in 2017, 37 in 2018, 66 in 2020 and 25 in just the first quarter of 2021 . The marked increase in 2020 and 2021 in these presentations is clearly evident. Ougrin et al. published data from 10 countries, reporting a rapid rise and adolescent self-harm presentation in ED during the current pandemic. ${ }^{4}$

Deliberate self harm, suicidal ideation, substance misuse, panic attack, generalised anxiety and low mood were the common presentations with which most of our adolescent patients presented to ED. This pattern has been consistent with emergency presentations of this age group in other parts of the world, ${ }^{3,5}$ but the recent and rapid rise in one of the busiest hospitals in Ireland is of great concern. There is a clear rise in ED psychiatric presentations of this age group, which may or may not be in the context of axis I psychiatric conditions; and may be a reflection of poor adjustment to the current pandemic. Due to the acute nature of assessment in the ED and lack of electronic patient record, reliable diagnoses and information are rarely available. ${ }^{3}$

The direct and indirect impact of COVID-19 on young people, together with compromised primary care counselling and mental health services, may be some of the important reasons behind the current rise in these presentations. Educational institutions and work places have been closed or restricted to online interaction also. The coping mechanisms in these stressful times for some of this vulnerable age group seems to be due to increased use of drugs, alcohol and self-harm. There is concern for both short term and long term mental health problems, which may warrant engagement with CAMHS and later adult psychiatric services. Policy making regarding psychological adjustment to this new-normal with the assistance of parents and teachers, generating healthy activities, running family physician and counselling services effectively and improving resources for CAMHS in these difficult times may be helpful in prevention or early diagnosis for mental health problems among theyoung population of Ireland.

\section{CONFLICT OF INTEREST:}

The authors declared no conflict of interest.

\section{AUTHORS' CONTRIBUTION:}

UBZ: Conceived the idea, worked with patients. wrote manuscript. EM, NB: Managed data, proof read manuscript.

\section{REFERENCES}

1. Fombonne E, Wostear G, Cooper V, Harrington R, Rutter M. The Maudsley long-term follow-up of child and adolescent depression. 2. Suicidality, criminality and social dysfunction in adulthood. Br J Psychiatry 2001; 179:218-23. doi: 10.1192/bjp.179.3.218.

2. Choi TK, Worley MJ, Trim RS, Howard D, Brown SA, Hopfer $\mathrm{CJ}$, et al. Effect of adolescent substance use and antisocial behavior on the development of early adulthood depression. Psychiatry Res 2016; 238:143-9. doi: 10.1016/j.psychres.2016.02.036.

3. Maguire E, Glynn K, McGrath C, Byrne P. Children, seen and heard: A descriptive study of all children (aged 12 years and under) referred for acute psychiatric assessment in Tallaght University Hospital over a 10-year period. Ir J Psychol Med 2020; 1-9. doi: 10.1017/ipm.2020.85.

4. Ougrin D, Wong BH, Vaezinejad M. Pandemic-related emergency psychiatric presentations for self-harm of children and adolescents in 10 countries (PREP-kids): A retrospective international cohort study. Eur Child Adolesc Psychiatry 2021; 1-13. doi:10.1007/s00787-021-01741-6

5. Kalb LG, Stapp EK, Ballard ED, Holingue C, Keefer A, Riley A. Trends in psychiatric emergency department visits among youth and young adults in the US. Pediatrics 2019; 143(4):e20182192. doi: 10.1542/peds.2018-2192.

Usama Bin Zubair, Erica Maguire and Noreen Bannan

Department of Psychiatry, Conolly Hospital, Dublin, Ireland

Correspondence to: Dr. Usama Bin Zubair, Department of Psychiatry, Conolly Hospital, Dublin, Ireland

E-mail: drusamabinzubair@yahoo.com

Received: May 11, 2021; Revised: May 27, 2021;

Accepted: June 23, 2021

DOI: https://doi.org/10.29271/jcpsp.2022.03.414 